\title{
A study of sexual abuse in an Asian community
}

\author{
Nadeem E Moghal, Inder K Nota, Christopher J Hobbs
}

\begin{abstract}
Sexual abuse in Asian ethnic minority populations in the UK has not previously been investigated. There remain professionals as well as lay people who deny its existence on the basis of assumptions about culture and religion. This retrospective study highlights the existence and difference in the incidence, pattern of presentation, and management in cases of Asian sexual abuse compared with that reported on the indigenous population. (Arch Dis Child 1995; 72: 346-347)
\end{abstract}

Keywords: sexual abuse, Asian.

Child sexual abuse is an area of paediatric medicine that has grown in recent times. ${ }^{1}$ The UK has a sizeable ethnic minority population. Leeds has Pakistani, Bangladeshi, and Indian populations that comprise $3 \%$ of the total population. ${ }^{2}$ Although cases of sexual abuse of Asian children are occasionally recognised, the existence of the problem itself is still denied by lay members of the public as well as some child welfare professionals. The aim of this study was firstly to test the hypothesis that sexual abuse does not occur because of the 'nature of Asian family structure' when compared with indigenous British populations. The study also aimed to look at the reported incidence, modes of presentation, management, and outcome of the cases and to compare the findings with previous studies. ${ }^{3} 4$

\section{Methods}

This was a retrospective study identifying cases of definite or probable sexual abuse referred to the two teaching hospitals in Leeds (St James's University Hospital and Leeds General Infirmary) over an eight year period (1985-93). The definition of definite or probable sexual abuse used was based on the description outlined in previous studies. ${ }^{34}$ Referrals came from hospital departments and community agencies of which social services was the principal one. The area covered was the city of Leeds.

St James's University Hospital, Leeds N E Moghal I K Nota

C J Hobbs

Correspondence to: Dr Nadeem E Moghal, Academic Unit of Paediatrics, Clinical Sciences Building, St James's Building, St James's University

Accepted 19 January 1995

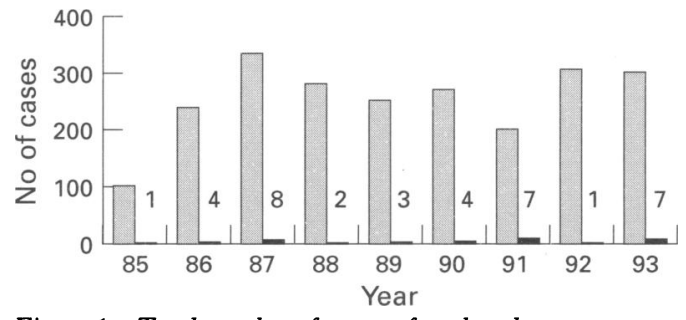

Figure 1 Total number of cases referred each year (1985-93). Number of Asian children adjacent to column.
Using medical reports, children of Asian origin were identified on the basis of name. Children of mixed race were excluded from the study. The ethnic minorities studied were those of Pakistani, Bangladeshi and Indian origin, the largest Asian population in Leeds. The 1991 census data ${ }^{2}$ were used to calculate incidence rates. A comparison of pattern of presentation and physical findings was made with data described by Frothingham et al, who undertook a retrospective review of children who suffered definite or probable sexual abuse referred to the same two hospitals in Leeds. ${ }^{4}$ The cohort included three children of Asian origin identified in this study.

\section{Results}

Thirty seven children were identified over the eight year period. The age range was 1-15 years (mean 7 years, mode 3 years) with 25 girls and 12 boys.

Figure 1 shows the yearly referral rate of cases of definite or probable sexual abuse to the two hospitals. Using census data we calculated the mean number of cases diagnosed by paediatricians per child population (0-15 years) per year; this was $0 \cdot 2 \%$ in non-Asians and $0.05 \%$ in Asians.

The predominant referral agency to paediatricians was social services (21), with others from family members (5), general practitioners (3), school (1), police (1), and after hospital admissions of children admitted for 'medical' or 'surgical' symptoms where abuse was the underlying cause (6).

The Asian children presented predominantly with genital/anal signs and symptoms $(43 \%)$, behaviour disturbances $(30 \%)$, nonspecific/psychosomatic signs and symptoms $(30 \%)$, and disclosure $(27 \%)$. The other modes of presentation included carer suspected abuse $(8 \%)$, sibling in contact with perpetrator $(8 \%)$, physical abuse $(3 \%)$, and perpetrator confession (3\%). Figure 2 displays the pattern of presentation and compares it against the 1989 cohort as described by Frothingham et al. ${ }^{4}$

Physical examination of the anus and genitalia identified signs of injury in 27 of the 37 children $(73 \%)$. Seven children had signs of physical abuse which included unexplained bruising, scald marks and cigarette burns; two children were failing to thrive.

Perpetrators were identified in 16 of the 37 cases. All of the perpetrators were male; 13 were of Asian origin. The majority of the perpetrators were known to their victims: father (7), mother's partner (3), uncle (1), grandfather (1), brother (1); three were strangers. Although three were charged with offences 


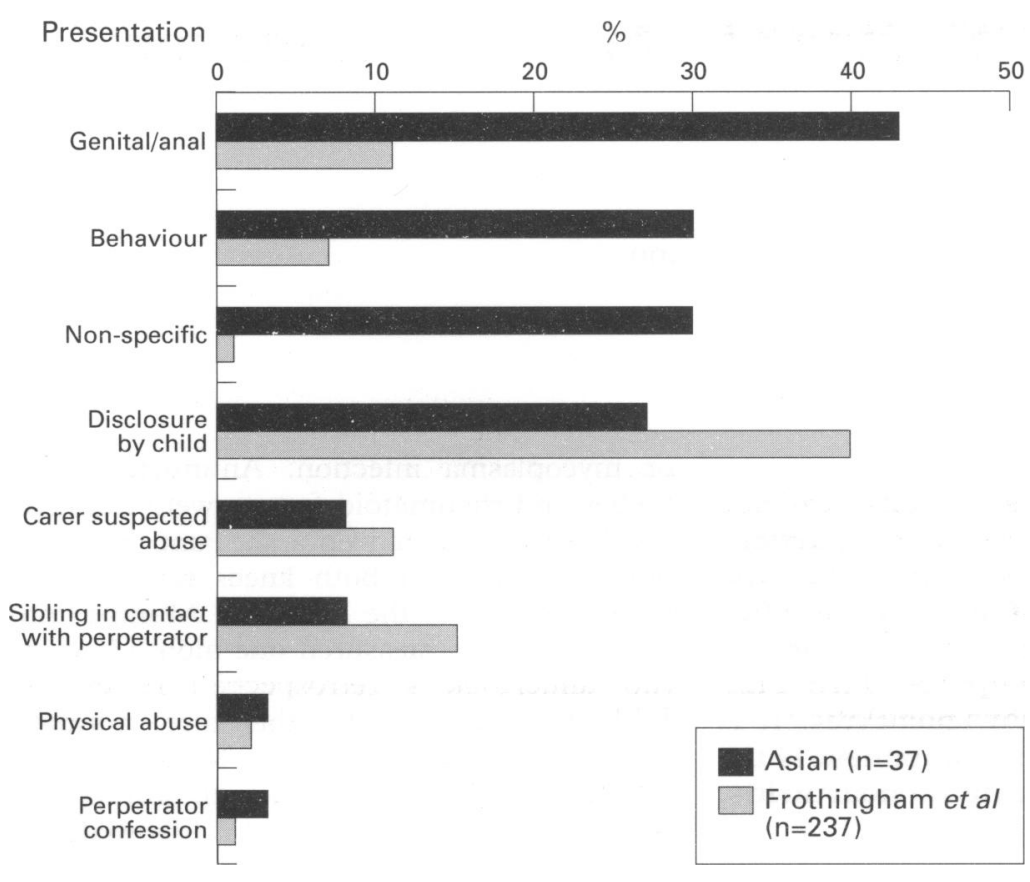

Figure 2 Pattern of presentation. structure, with emphasis placed on religion and culture, is not supported by these findings.

While the age distribution and gender ratio is similar to previous reports, ${ }^{3}$ the incidence of cases diagnosed in the two hospitals is lower than the overall incidence in the population. Cases appear to be more severe and probably represent the tip of a larger iceberg.

Apart from genital/anal signs and symptoms, behaviour disturbance and non-specific signs and symptoms were predominant modes of presentation. Asian children were therefore more likely to present in ways in which the professional needed a higher index of suspicion regarding the possibility of sexual abuse. As a cohort the Asian children disclosed less frequently and family members were less likely to initiate concerns when compared with the Frothingham cohort (fig 2). ${ }^{4}$

We do not know the reasons for the almost complete lack of Asian professional involvement, including interpreters and link workers. However, we believe these professionals would have much to contribute. Owing to their small numbers within child welfare agencies a positive decision is needed to recruit them to help with particular cases.

The perpetrators identified were all men. Child sexual abuse by women is now recognised, ${ }^{5}$ reflecting increasing awareness and understanding of the problem. There is no reason to doubt that women perpetrators also exist within the Asian communities. The low conviction rate for sexual abuse reflects the current situation in the population as a whole.

The difference in those reaching case conference and being registered as sexual abuse between the Asian and Frothingham cohort suggests that in the former evidence had to be overwhelming before a case conference was held. Professionals may be less keen to act where Asian children are concerned unless they are sure of their facts.

The effects of sexual abuse on a child's physical and mental health should not be underestimated and the nature and severity of the effects are unlikely to be related to the ethnic background. However, ethnicity has implications in the identification and management of these children.

We thank D C I Harrington of the Domestic Violence and Child Abuse Unit, Wakefield and Mrs C Lees, administrator for the child protection register, Leeds social services for helping with data collection.

1 Hobbs CJ, Hanks HGI, Wynne JM. Child abuse and neglect. Edinburgh: Churchill Livingstone, 1994

2 Office of Population Censuses and Surveys. 1991 Census of population Great Britain, local base statistics for Leeds. London: HMSO Publications, 1993.

\section{Discussion}

This study was prompted by the almost complete lack of information on the subject of child sexual abuse in Asian minority populations in the UK. The hypothesis that sexual abuse does not occur because of the Asian family 